Published in final edited form as:

Epileptic Disord. 2009 September ; 11(3): 206-214. doi:10.1684/epd.2009.0273.

\title{
Basic mechanisms of MCD in animal models
}

\section{Giorgio Battaglia ${ }^{1}$, Albert J. Becker², Joseph LoTurco ${ }^{3}$, Alfonso Represa ${ }^{4}$, Scott C. Baraban $^{5}$, Steven N. Roper ${ }^{6}$, and Annamaria Vezzani ${ }^{7}$}

${ }^{1}$ Molecular Neuroanatomy and Pathogenesis Unit, IRCCS Foundation Neurological Institute

"Carlo Besta", Milano, Italy

${ }^{2}$ Department of Neuropathology, University of Bonn Medical Center, Bonn, Germany

${ }^{3}$ Department of Physiology and Neurobiology, University of Connecticut, Storrs CT, USA

${ }^{4}$ INMED, Inserm U901, Université de la Méditerranée, Campus de Luminy, Marseille, France

${ }^{5}$ Department of Neurological Surgery, University of California, San Francisco, CA, USA

${ }^{6}$ Department of Neurological Surgery, McKnight Brain Institute, University of Florida, Gainesville, Florida, USA

${ }^{7}$ Department of Neuroscience, Mario Negri Institute for Pharmacological Research, Milano, Italy

\section{Abstract}

\begin{abstract}
Epilepsy-associated glioneuronal malformations (malformations of cortical development [MCD]) include focal cortical dysplasias (FCD) and highly differentiated glioneuronal tumors, most frequently gangliogliomas. The neuropathological findings are variable but suggest aberrant proliferation, migration, and differentiation of neural precursor cells as essential pathogenetic elements. Recent advances in animal models for MCDs allow new insights in the molecular pathogenesis of these epilepsy-associated lesions. Novel approaches, presented here, comprise RNA interference strategies to generate and study experimental models of subcortical band heterotopia and study functional aspects of aberrantly shaped and positioned neurons. Exciting analyses address impaired NMDA receptor expression in FCD animal models compared to human FCDs and excitatory imbalances in MCD animal models such as lissencephaly gene ablated mice as well as in utero irradiated rats. An improved understanding of relevant pathomechanisms will advance the development of targeted treatment strategies for epilepsy-associated malformations.
\end{abstract}

\section{Keywords}

epileptogenesis; glutamate receptors; methylazoxymethanol; pilocarpine; in utero irradiation; MAEUK proteins; MCD; animal model

\footnotetext{
Correspondence: A. Vezzani, Lab. Experimental Neurology, Dept of Neuroscience, Mario Negri Inst for Pharmacol Res, Via G. La Masa 19, 20156 Milano, Italy, <vezzani@marionegri.it>.

Presented at the "Malformations of Cortical Development and Epilepsy" Symposium, organized in Istanbul, Turkey (October 30-31, 2008) by the ILAE Commissions of Neurobiology and Therapeutic strategies together with the Cerrahpasa Medical Faculty of Istanbul University.

Disclosures.

None.
} 
Circumscribed malformative lesions of the CNS comprise a wide spectrum of neuroradiological and histomorphological alterations that are an important cause of developmental disabilities and focal epilepsy in humans. They are clinically as well as histologically diverse, ranging from subtle architectural aberrations to highly dysplastic lesions with respect to cytological and structural characteristics. Present classifications of malformations of cortical development (MCDs) are based on histological features such as loss of cortical lamination, glio-neuronal and/or neuronal heterotopias, the occurrence of dysplastic or cytomegalic neuronal elements and dysmorphic glial cell elements, so-called "Taylor-type" balloon cells. Although the described cytological and histological alterations generally relate MCDs to compromised migration and differentiation of neuronal precursors during cortex development, their pathogenetic basis and mechanisms of epileptogenicity are only incompletely understood.

For many years, a severe obstacle was presented by the fact that appropriate animal models of these highly differentiated malformations were not available. The present paper summarizes the essential of the presentations and discussions during the basic science session of the "Malformations of Cortical Development and Epilepsy" Symposium. The speakers opened new avenues to overcome the problem of lack of clinically relevant animal models along with three different research approaches. First, the use of RNA interference through gene transfer strategies in vivo; second, the close morphological and molecular comparisons between newly developed models and human tissue samples from patients suffering from pharmacorefractory epilepsy; third, the neurophysiological analysis of the balance between excitation and inhibition in the malformed and dysplastic cortex. Thus, the speakers have presented a kaleidoscope of fascinating approaches with the final goal of unravelling molecular mechanisms underlying the development of cortical malformations, as well as cellular and subcellular alterations that promote epileptogenicity of these lesions.

\section{The use of RNA interference to generate and study experimental models of subcortical band heterotopia}

The first two presentations by Lo Turco and Represa describe experimental models of subcortical band heterotopia (SBH). In human patients, SBH is a cortical malformation characterized by the presence of large clusters of neurons within the white matter underlying a relatively normal cortex, determining the so-called "double cortex". This condition has been reported in a woman with mutation of the $D C X$ gene, and in patients with mutations of TUBAIA and LIS1 genes (Guerrini et al., 2008) but other genes are most likely involved.

SBH is associated with mental retardation and intractable infantile epilepsies. The available animal models of band heterotopia are impinged by intrinsic limitations. $\mathrm{DCX}^{-1-}$ and $\mathrm{LIS1}^{-/-}$mice have been generated but they display no neocortical abnormality, therefore lessening their impact on the field (Cahana et al., 2001; Corbo et al., 2002; Hirotsune et al., 1998). An interesting model of band heterotopia is the TISH mutant rat (Schottler et al., 1998); however, the gene responsible for this phenotype remains unknown and the phenotype involves quite different molecular and cellular mechanisms. More recently a similar "orphan" model of band heterotopia, HeCo mutant mice, has been reported (Croquelois et al., 2008), but the model has not been yet fully characterized. Consequently, 
we have still little information on the neurobiology of SBH and the cellular and network properties of neurons within the "double cortex".

In contrast, the in utero knock-down of DCX RNA produces a morphologically relevant cortical band heterotopia in rodents (Bai et al., 2003) providing an excellent tool to investigate this issue.

The group of LoTurco addresses the issue of whether neocortical malformations and associated functional impairments can be reversed by reactivating developmental programs in the developing brain once malformations are detected. They rely on an animal model of SBH in which RNA interference (RNAi) is first used to induce the brain malformation, then conditional re-expression of the targeted gene is used to restart migration after the malformation has already formed (Manent et al., 2009; figure 1). The authors found that it is possible to re-start migration in the early postnatal rat brain, and this reactivation of migration reduces the size of SBH and restores neuronal patterning. They observed further that the capacity to regress heterotopia and restore normal migration patterns shows a critical period in early postnatal development. Moreover, reactivation of migration reduces seizure threshold to injection of PTZ to levels similar to that of malformation-free control animals. These results suggest that future approaches directed at restarting migration during a critical period of development may reduce the risk of seizure development in malformed cerebral neocortex.

The group of Represa relies on the same RNAi preparation to compare the neuronal and network properties of heterotopic neurons, cortical neurons overlying the heterotopia and control neurons to identify how heterotopic neurons generate adverse patterns that will impact cortical activity (Ackman et al., 2009; figure 2). By combining dynamic calcium imaging and anatomo-electrophysiological techniques, they demonstrate that $\mathrm{DCX}^{-/} \mathrm{EGFP}^{+}$ labelled heterotopic neurons that fail to migrate, develop extensive axonal subcortical projections, retain immature properties and display a dramatic absence of GABA-mediated signalling (Ackman et al., 2009). Cortical neurons overlaying the heterotopia exhibit, in contrast, a massive increase of ongoing glutamatergic synaptic currents reflecting a strong reactive plasticity. Neurons in both experimental fields are more frequently coactive in coherent synchronized oscillations than control cortical neurons. In addition, both fields display network-driven oscillations during evoked epileptiform burst. Represa reported preliminary data supporting the notion that epileptiform activities initiate from neurons on the cortex overlaying the heterotopia and involve the heterotopic neurons in a relatively synchronized way (Ackman et al., 2009). These results show that DCX cortex involves major alterations not only in neurons that fail to migrate but also in their programmed target areas. Based on these observations, the author suggests that this duality plays a major role in cortical dysfunction of DCX brains.

These observations are in agreement with previous reports from human patients depicting BOLD changes on EEG-fMRI analysis during ictal and interictal events in both fields (Kobayashi et al., 2006). The analysis of other models of brain malformation, such as MAM- or $\gamma$-ray-treated rats (Colacitti et al., 1999; Roper, 1998), suggests similarly that ectopic neurons are not epileptogenic per se but they participate in the epileptiform activities 
generated by convulsant agents or protocols, through their strategic position at the frontier of two different fields, the hippocampus and the somatosensory cortex. Indeed, ectopic neurons in MAM rats are neurons of cortical origin that maintain their cortical connections while acquiring within the hippocampus, inputs from hippocampal pyramidal neurons (ChevassusAu-Louis et al., 1998). In this model, activities generated within the hippocampus will transit thorough the heterotopia to reach the neocortex. Intrahippocampal heterotopias play therefore a capital role in the propagation but not the genesis of abnormal activities.

In conclusion, Represa et al. propose that the genesis of heterotopia combined with the subsequent alteration of cortical networks involving the overlaying neocortex could have important consequences for cortical function and would be involved in the neurological manifestations linked to this type of disorder, such as mental delay and childhood epilepsy.

\section{Morphological and molecular comparison between human patients and experimental models of focal cortical dysplasia}

Battaglia et al. are currently investigating the NMDA receptor complex and related protein composition in focal cortical dysplasia (FCD), which is the more common type of MCD in human patients, characterized by a severe epilepsy course, often requiring epilepsy surgery for seizure control. They have used the same combined morphological and molecular approach to investigate possible molecular mechanisms of epileptogenesis shared by both human patients and experimental models. They have analyzed epileptogenic cortical samples from human patients affected by type IA and IIB FCD obtained during epilepsy surgery. The experimental counterpart is provided by a "double-hit" rat model, generated by inducing brain malformations with prenatal exposure to methylazoxymethanol (MAM) and spontaneous recurrent seizures induced by post-natal pilocarpine treatment in MAM-treated adult rats. Their results clearly indicate the presence of NMDA receptor complex abnormalities in both type IA and IIB focal cortical dysplasia. In type IA FCDs, a selective increase in the regulatory subunit NR2B was present in all considered cases. NR2B upregulation was greater in the total homogenate than the post-synaptic membrane fraction, suggesting that mechanisms other than increased ionic influx through the post-synaptic membrane may sustain hyperexcitability in dysplastic neurons (Finardi et al., 2006; figure 3). By contrast, in type IIB FCDs, a more intense NR2B staining was particularly evident in the apical dendrites and enlarged dendritic spines of giant pyramidal neurons. Accordingly, the molecular analysis showed a significant and selective increase of NR2A/B subunits and their scaffolding proteins, SAP97 and PSD95, in the post-synaptic membrane compartments. Since this up-regulation was not accompanied by an increased activation state of CREB and ERK1/2, it may reflect the presence of excessive extra-synaptic NMDA receptors, which may trigger intracellular pathways in the giant dysplastic cortical neurons underlying their pathological features.

In the analysis of the double-hit MAM/pilocarpine model, the authors observed that the onset of spontaneous seizures was anticipated and that seizure number and frequency was increased when compared to control rats treated with pilocarpine. These data confirm that in the MAM model the presence of brain malformations reduces the threshold to proconvulsant stimuli. In addition, the histological and molecular analysis of fractions of 
cortical malformed tissue enriched in post-synaptic densities, revealed a clear-cut increase in the expression level of the NMDA regulatory subunits in the chronic phase of epilepsy, with a consistent recruitment of the same subunits in the apical dendrites of large and dysplastic pyramidal neurons. The NR2A/B up-regulation was associated with increased cell size and expression of cytoskeletal elements, similarly to what was observed in giant dysplastic pyramidal neurons of human FCD IIB patients Taken together, data collected both from human MCDs tissues and MAM-pilocarpine treated rats support the existence of a relevant plasticity of the NMDA receptor complex, which could play a role in the basic mechanisms of hyperexcitability associated with MCDs. Moreover, they indicate that MAM/pilocarpine rats represent a suitable double-hit model to study the pathogenic mechanisms of epilepsy and possibly the pathological neuronal plasticity associated with repeated seizures in human MCDs.

\section{Altered balance between excitation and inhibition in different animal models of MCDs}

The last two speakers of the basic science session, Baraban and Roper utilize similar neurophysiological approaches to investigate the balance between excitatory and inhibitory inputs in two different animal models, the $\mathrm{Lis}^{+/-}$mice and the in utero irradiated rats.

It is well known from human studies that a heterozygous mutation or deletion of the lissencephaly gene Lis 1 is associated with severe disruption of cortical and hippocampal lamination, cognitive deficit and severe seizures. Mice with one null allele of Lis1 (Lis $1^{+/-}$ mice) exhibit significant hippocampal malformations and slowed migration of interneuron precursors. In addition, preliminary video-EEG data demonstrated spontaneous electrographic seizures in some of these mice.

Baraban et al. first analyzed glutamate-mediated synaptic excitation of this model. Isolated whole-cell excitatory postsynaptic current (EPSC) on visually identified pyramidal neurons in disorganized CA1 regions of hippocampal slices prepared from $\mathrm{Lis}^{+/-}$mice were recorded. They observed a two-fold increase in spontaneous and miniature EPSC frequency with no change in amplitude or decay kinetics, and using paired-pulse and $10 \mathrm{~Hz}$ stimulation protocols, a dramatic increase in presynaptic excitation. Deficits in paired-pulse facilitation could be restored to wild-type levels with the addition of cadmium chloride, a voltageactivated calcium channel blocker. Electron microscopy analysis revealed a significant increase in synaptic vesicle density with no change in the size of the active zone or terminal area. Taken together, this first set of experiments suggests the existence of an excitable and pro-epileptic circuit in the malformed hippocampus of Lis1 mutant mice.

Second, they analyzed GABA-mediated synaptic inhibition by recording isolated whole cell inhibitory postsynaptic currents (IPSCs) on visually identified CA1 pyramidal neurons in slices prepared from Lis $1^{+/-}$mice. They observed a $32 \%$ increase in spontaneous IPSC frequency in Lis $1^{+/-}$mice compared with normotopic CA1 pyramidal neurons in agematched controls. This increase was not associated with a change in spontaneous IPSC decay or miniature IPSC frequency. Mean IPSC amplitude was increased, and event histograms indicated a greater number of large (>125 pA) events. Tonic inhibition, response 
to paired-pulse stimulation and evoked IPSC decay kinetics were not altered. Consistent with increased synaptic inhibition, Lis $1^{+/-}$interneurons also exhibited more spontaneous firing in cell-attached recordings and increased excitation as measured by voltage-clamp recording of spontaneous excitatory postsynaptic currents (EPSCs) onto interneurons (Jones and Baraban, 2007; figure 4). This up-regulation of inhibition may be a compensatory response to the increased excitation and hyperexcitability observed in these animals.

In addition to neurophysiological abnormalities, the authors also observed a significant disruption of the subgranular zone and glial fibrillary acidic protein-immunoreactive radial astrocytes in the dentate gyrus of adult LIS1 mice. Using pulse-chase bromo-deoxyuridine (BrdU) labeling combined with neuronal and glial antibody staining, they provide evidence for ectopic adult neurogenesis in LIS1 mice. A gradually decreased survival rate for these newborn granule cells was also demonstrated in LIS1 mice seven days after BrdU injection. This reduced survival rate was associated with impaired neuronal differentiation 28 days after BrdU administration. Thus, LIS1 haploinsufficiency can lead to abnormal cell proliferation, migration and differentiation in the adult dentate gyrus.

A similar approach was undertaken by Roper and colleagues using a different model, the in utero irradiated rats (Chen et al., 2007; figure 5). This model was first described to study abnormal brain development (Cowan and Geller, 1960) but epilepsy was not a focus of these early investigations. The protocol involves exposure of a pregnant dam to external radiation (225 cGy of radiation from a linear accelerator source) on the 17th day of gestation (E17). The offspring are born on E21 and weaned on P21. Cortical malformation in this model is the result of two related processes: cellular damage from the initial radiation injury and continued addition of new cells to the neocortex after injury. The initial damage is extensive and necrosis can be seen throughout the neocortex, particularly in the intermediate layer, with relative sparing of progenitor cells in the ventricular zone and neurons already migrated into the cortical plate. There is some evidence that migrating, immature neurons are the most susceptible to localise at the radiation injury (Altman et al., 1968). Histologically, treated animals are microcephalic, with loss of lamination, abnormal spatial orientation of neurons, periventricular and subcortical heterotopia, focal areas of heterotopic neurons in the hippocampus and hypoplasia or agenesis of the corpus callosum. Neither giant dysmorphic neurons nor balloon cells were ever described. Therefore, in utero irradiation is an injurybased model of diffuse cortical malfomation similar to type I FCD, according to the Palmini classification system (Palmini et al., 2004). Spontaneous seizures have been previously described in these animals (Kellinghaus et al., 2004).

In this model, a striking feature reported by the authors is the impaired development of the inhibitory system in the dysplastic cortex, demonstrated by using both histological and physiological methods. In early experiments performed in adult irradiated and control animals, two-dimensional cell counting data showed a 50\% reduction in the density of parvalbumin (PA) and calbindin D28k (CB) immunoreactive interneurons with no reduction in total neuronal density (Roper et al., 1999). This evidence suggests a relative reduction in the number of inhibitory interneurons in the dysplastic cortex. It should be underlined that a relative loss of cortical interneurons was also reported in studies from human FCD patients (Calcagnotto et al., 2005). 
They have also evaluated functional connectivity in dysplastic cortex using spontaneously occurring post-synaptic currents (PSCs) (Zhu and Roper, 2000). Using whole cell patch clamp methods, they recorded excitatory and inhibitory PSCs (EPSCs and IPSCs) from large pyramidal cells in irradiated and control neocortex. They found a reduction in the frequency of spontaneous and miniature (post-tetrodotoxin) IPSCs. Conversely, spontaneous EPSCs had an increased frequency in dysplastic cortex, and miniature EPSCs were no different from controls. Consistent with the immunohistochemical findings, this evidence suggests a reduction in inhibitory connections as compared to excitatory contacts in the irradiated cortex. Identical findings were obtained in the subcortical heterotopic grey matter of irradiated rats (Chen and Roper, 2003).

The authors also recorded EPSCs from surviving interneurons in the irradiated rats identifying two types of interneurons on the basis of their physiological properties (Xiang et al., 2006): fast-spiking with short-term depression (type I) and regular-spiking with shortterm facilitation (type II) interneurons. Both types of interneurons showed a reduced frequency of EPSCs in dysplastic cortex, therefore suggesting the existence of a reduced excitatory drive on the surviving interneurons in this model.

The authors also addressed the issue of short-term plasticity (STP), which reflects dynamic function of cortical synapses and exerts relevant effects on the neuronal circuitry. In normal layer $\mathrm{V}$ pyramidal cells, repetitive activation of pre-synaptic axons results in short-term depression of EPSCs at age two weeks, then switching to short-term facilitation at age four weeks (Reyes and Sakmann, 1999). This developmental switch involves increased activation of pre-synaptic type 2/3 metabotropic glutamate receptors (Chen and Roper, 2004), most likely resulting from increased extracellular glutamate concentrations during this age period. In irradiated rats, the normal switch to short-term facilitation did not occur in cortical pyramidal neurons (Chen and Roper, 2007). Indeed, these animals demonstrated the persistence of short-term depression at two and four weeks, and in adulthood. This suggests an abnormal persistence of increased release probability in the excitatory pre-synaptic terminals synapsing on large pyramidal cells, therefore potentially leading to increased excitability of the dysplastic cortex.

In summary, both groups have demonstrated a number of abnormalities in the balance between inhibition and excitation in the malformed cortical circuitry in the two models of genetic and acquired cortical malformations which may be relevant to increase the propensity for epileptiform activity.

\section{Conclusion}

The development and characterization of clinically relevant models of cortical malformations is a major challenge for basic science to study the mechanisms underlying aberrant neuronal migration and the associated epileptic phenotype. The basic science session held at "Malformations of Cortical Development and Epilepsy" Symposium has provided a multidisciplinary approach to this issue: the use of modern molecular biology techniques associated with the close comparison at morphological, molecular and neurophysiological levels, between experimental and human malformed cortical samples. A 
major task remains to carefully characterize the epileptic phenotype, or decreased seizure threshold, in the genetically determined acquired models of cortical malformations. These research efforts are instrumental to increase our understanding of the pathogenetic mechanisms and to envisage novel targets and effective therapeutic approaches for treating epilepsy in human MCD patients.

\section{References}

Ackman JB, Aniksztejn L, Crépel V, et al. Abnormal Network Activity in a Targeted Genetic Model of Human Double Cortex. J Neurosci. 2009; 29:313-327. [PubMed: 19144832]

Altman J, Anderson WJ, Wright KA. Differential radiosensitivity of stationary and migratory primitive cells in the brain of infant rats. Exp Neurol. 1968; 22:52-74. [PubMed: 5687685]

Bai J, Ramos RL, Ackman JB, et al. RNAi reveals doublecortin is required for radial migration in rat neocortex. Nat Neurosci. 2003; 6:1277-1283. [PubMed: 14625554]

Cahana A, Escamez T, Nowakowski RS, et al. Targeted mutagenesis of Lis1 disrupts cortical development and LIS1 homodimerization. Proc Natl Acad Sci USA. 2001; 98:6429-6434. [PubMed: 11344260]

Calcagnotto ME, Parades MF, Tihan T, et al. Dysfunction of synaptic inhibition in epilepsy associated with focal cortical dysplasia. J Neurosci. 2005; 25:9649-9657. [PubMed: 16237169]

Chen HX, Roper SN. Reduction of spontaneous inhibitory synaptic activity in experimental heterotopic gray matter. J Neurophysiol. 2003; 89:150-158. [PubMed: 12522167]

Chen HX, Roper SN. Tonic activity of metabotropic glutamate receptors is involved in developmental modification of short-term plasticity in the neocortex. J Neurophysiol. 2004; 92:838-844. [PubMed: 15044523]

Chen HX, Xiang H, Roper SN. Impaired developmental switch of short-term plasticity in pyramidal cells of dysplastic cortex. Epilepsia. 2007; 48:141-148. [PubMed: 17241221]

Chevassus-Au-Louis N, Congar P, Represa A, et al. Neuronal migration disorders: heterotopic neocortical neurons in CA1 provide a bridge between the hippocampus and the neocortex. Proc Natl Acad Sci USA. 1998; 95:10263-10268. [PubMed: 9707635]

Colacitti C, Sancini G, DeBiasi S, et al. Prenatal methylazoxy-methanol treatment in rats produces brain abnormalities with morphological similarities to human developmental brain dysgeneses. J Neuropathol Exp Neurol. 1999; 58:92-106. [PubMed: 10068317]

Corbo JC, Deuel TA, Long JM, et al. Doublecortin is required in mice for lamination of the hippocampus but not the neocortex. J Neurosci. 2002; 22:7548-7557. [PubMed: 12196578]

Cowan D, Geller LM. Long-term pathological effects of prenatal X-irradiation on the central nervous system of the rat. J Neuropathol Exp Neurol. 1960; 19:488-527. [PubMed: 13696100]

Croquelois A, Giuliani F, Savary C, et al. Characterization of the HeCo Mutant Mouse: A New Model of Subcortical Band Heterotopia Associated with Seizures and Behavioral Deficits. Cereb Cortex. 2008; 19:563-575. [PubMed: 18562329]

Finardi A, Gardoni F, Bassanini S, et al. NMDA receptor composition differs among anatomically diverse malformations of cortical development. J Neuropathol Exp Neurol. 2006; 65:883-893. [PubMed: 16957582]

Guerrini R, Dobyns WB, Barkovich AJ. Abnormal development of the human cerebral cortex: genetics, functional consequences and treatment options. Trends Neurosci. 2008; 31:154-162. [PubMed: 18262290]

Hirotsune S, Fleck MW, Gambello MJ, et al. Graded reduction of Pafah1b1 (Lis1) activity results in neuronal migration defects and early embryonic lethality. Nature Gen. 1998; 19:333-339.

Jones DL, Baraban SC. Characterization of inhibitory circuits in the malformed hippocampus of Lis 1 mutant mice. J Neurophysiol. 2007; 98:2737-2746. [PubMed: 17881479]

Kellinghaus C, Kunieda T, Ying Z, et al. Severity of histopathologic abnormalities and in vivo epileptogenicity in the in utero radiation model of rats is dose dependent. Epilepsia. 2004; 45:583591. [PubMed: 15144422] 
Kobayashi E, Bagshaw AP, Grova C, et al. Grey matter heterotopia: what EEG-fMRI can tell us about epileptogenicity of neuronal migration disorders. Brain. 2006; 129:366-374. [PubMed: 16339793]

Manent JB, Wang Y, Chang Y, et al. Dcx reexpression reduces subcortical band heterotopia and seizure threshold in an animal model of neuronal migration disorder. Nat Med. 2009; 15:84-90. [PubMed: 19098909]

Palmini A, Najm I, Avanzini G, et al. Terminology and classification of the cortical dysplasias. Neurology. 2004; 62 Suppl. 3(6):52-58.

Reyes R, Sakmann B. Developmental switch in the short-term modification of unitary EPSPs evoked in Layer 2/3 and Layer 5 pyramidal neurons of rat neocortex. J Neurosci. 1999; 19:3827-3835. [PubMed: 10234015]

Roper SN, Eisenschenk S, King MA. Reduced density of parvalbumin- and calbindin D28kimmunoreactive neurons in experimental cortical dysplasia. Epilepsy Res. 1999; 37:63-71. [PubMed: 10515176]

Roper SN. In utero irradiation of rats as a model of human cerebrocortical dysgenesis: a review. Epilepsy Res. 1998; 32:63-74. [PubMed: 9761309]

Schottler F, Couture D, Rao A, et al. Subcortical connections of normotopic and heterotopic neurons in sensory and motor cortices of the tish mutant rat. J Comp Neurol. 1998; 395:29-42. [PubMed: 9590544]

Xiang H, Chen H-X, Yu X-X, et al. Reduced excitatory drive in interneurons in an animal model of cortical dysplasia. J Neurophysiol. 2006; 96:259-278. [PubMed: 16571734]

Zhu WJ, Roper SN. Reduced inhibition in an animal model of cortical dysplasia. J Neurosci. 2000; 20:8925-8931. [PubMed: 11102503] 


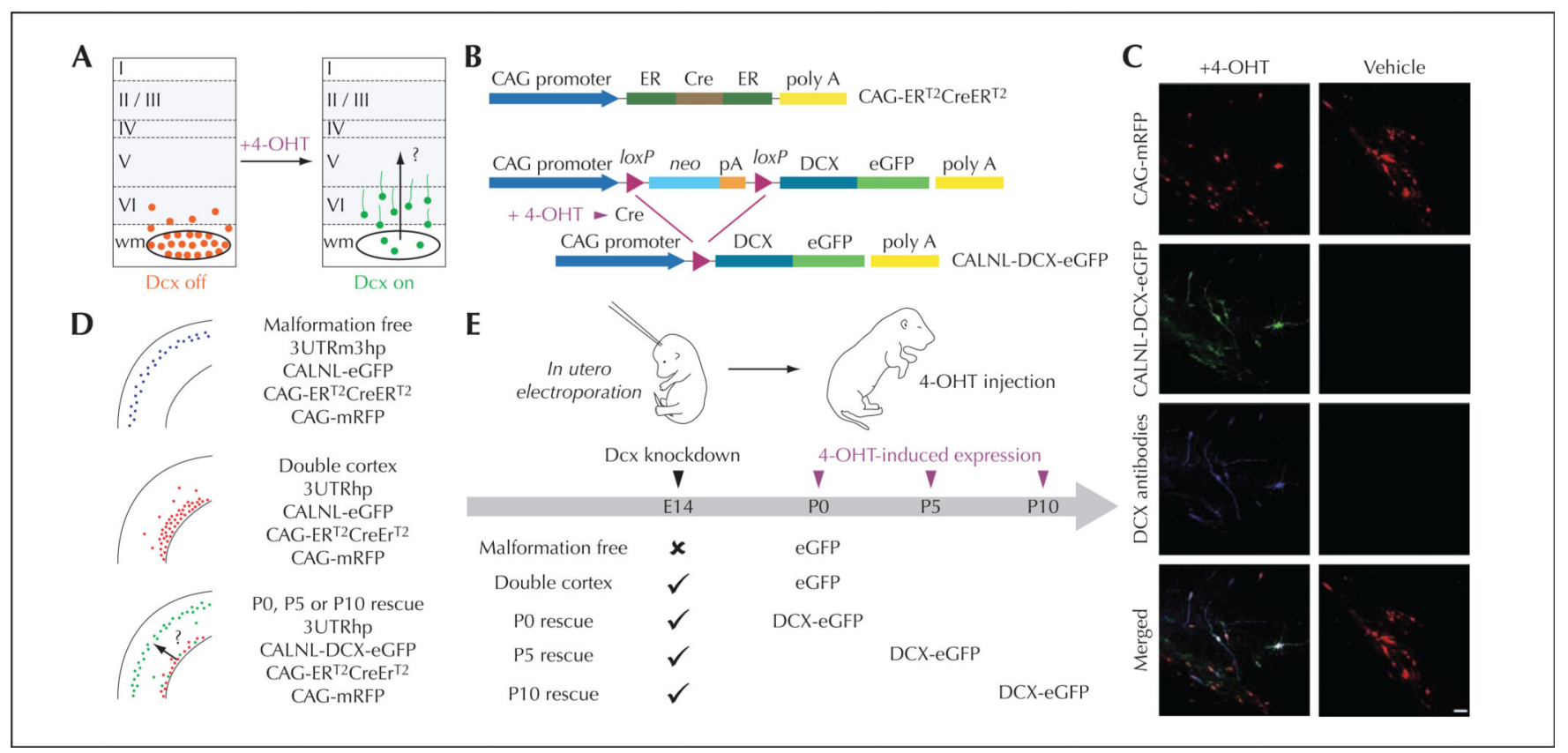

Figure 1.

Temporal control of Dcx expression in misplaced neurons by conditional re-expression of the Dcx gene (from Manent et al., 2009). A) A schematic diagram of the experimental approach to verify the hypothesis that re-expression of Dcx can re-start migration in the early postnatal rat brain, reduce the size of SBH and restore neuronal patterning. B) A 4hydrotamoxifen (4-OHT)-activatable Cre recombinase composed of two estrogen receptor (ER) binding domains is expressed under the control of the CAG promoter. In the presence of 4-OHT, recombination occurs and DCX-eGFP is expressed. C) Confocal images showing transfected neurons in neocortex of P15 rats that have received 4-OHT or vehicle injection at birth. Rats were electroporated at E14 with four plasmids. In 4-OHT-treated rats (left), DCX-GFP is expressed and Dcx is detectable with antibodies in transfected misplaced neurons. In vehicle-treated rats (right), no signal is detected in the green channel or with Dcx antibodies. D) Summary of the transfection conditions. E) Schematic diagram of experiments. Scale bar, $50 \mu \mathrm{m}$. 


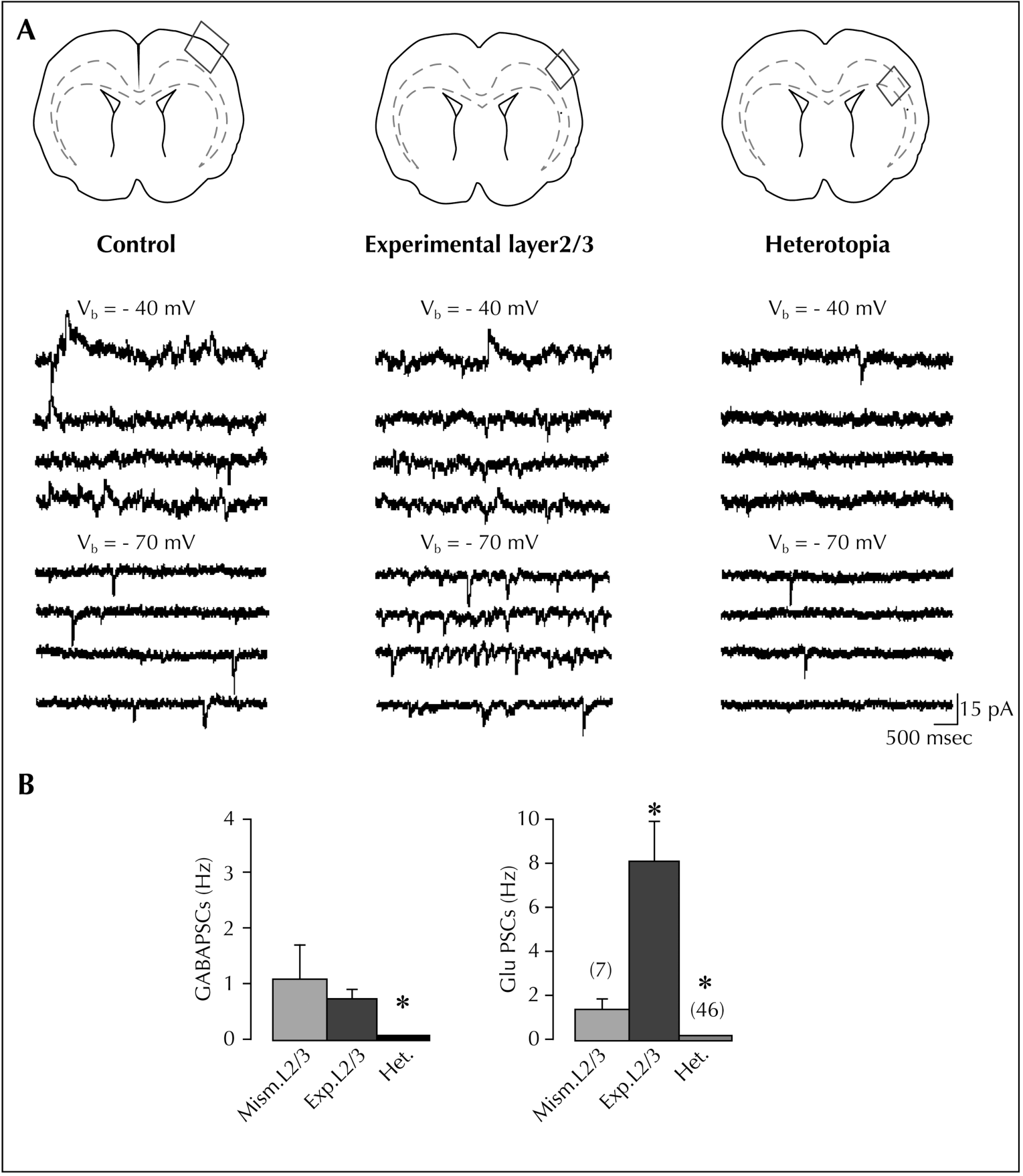

Figure 2.

Experimental L2/3 neurons display high synaptic glutamatergic drive (modified from Ackman et al., 2009): A) Example traces of ongoing spontaneous activity recorded at - 70 $\mathrm{mV}\left(\mathrm{ECl}^{-}\right)$and $-40 \mathrm{mV}$ in a pyramidal cell of control L2/3 (left traces), experimental L2/3 (middle traces), and white matter heterotopia (right traces). Schemes on the top indicate the field analyzed in each case. Note the large increase of glutamate PSCs in the pyramidal cell from experimental L2/3 compared with control. Note in the ectopic cell the low level of activity and the absence of GABA PSCs. B) Mean GABA (left) and glutamate (right) PSC 
frequencies in L2/3 pyramidal neurons from slices transfected with control mismatch (Mism) constructs or shDCX (experimental layers 2/3 and heterotopia). Note that the ongoing spontaneous activity in pyramidal cells from experimental L2/3 is largely dominated by glutamate. Most of the ectopic neurons (73\%) lack GABA events.

* Significantly different as compared to Mism.L2/3. 


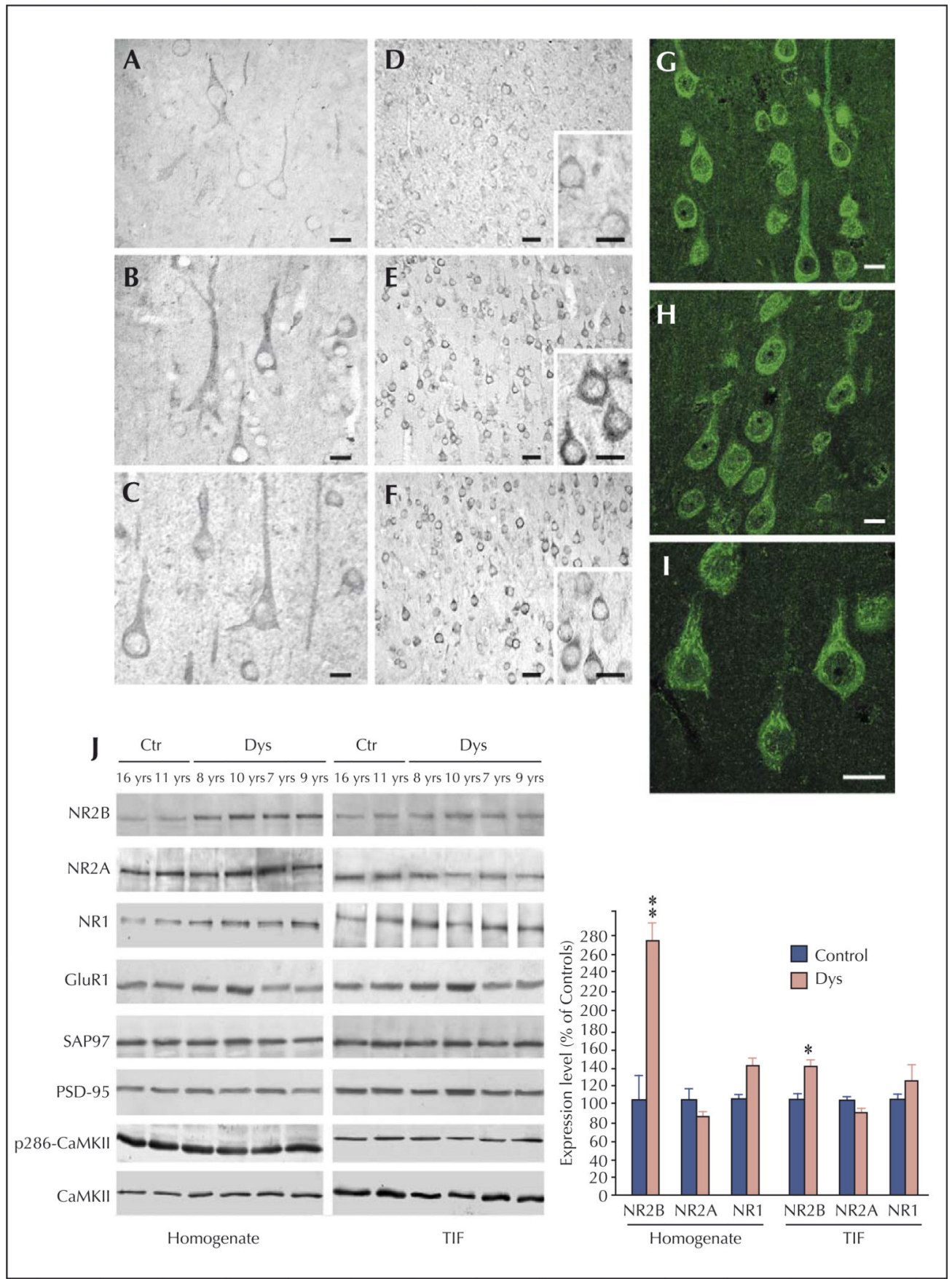

Figure 3.

NMDA receptor expression and composition are altered in type IA FCD patients (from Finardi et al., 2006). A-F) NR1 (A-C) and NR2B signals (D-F) are more evident in cortical pyramidal neurons from FCD patients $(\mathbf{B}-\mathbf{C}$ and $\mathbf{E}-\mathbf{F})$ in comparison with controls ( $\mathbf{A}$ and D). G-I) Confocal immunofluorescence confirmed the increased NR2B signal in coarse granules within the cell body and apical dendrites of FCD IA pyramidal neurons. J) Western blot analysis of homogenate (left) and TIF (right) fractions from cortical specimens of FCD patients (Dys) and controls (Ctr). Note that NR2B expression levels are increased in all FCD 
patients, whereas NR2A (patient 8, aged $7 \mathrm{yrs)} \mathrm{or} \mathrm{GluR1} \mathrm{(patient} \mathrm{7,} \mathrm{aged} 10 \mathrm{yrs)}$ expression levels are increased in some but not all patients. Statistical analysis reveals that NR2B was significantly increased in dysplastic versus control cortical areas $(* * \mathrm{p}<0.01$ for the homogenate; ${ }^{*} \mathrm{p}<0.05$ for post-synaptic membranes). Scale bars: $20 \mu \mathrm{m}$ (A-C); $50 \mu \mathrm{m}$ (D$\mathbf{F} ; 20 \mu \mathrm{m}$ in inserts); $20 \mu \mathrm{m}$ (G-I). 


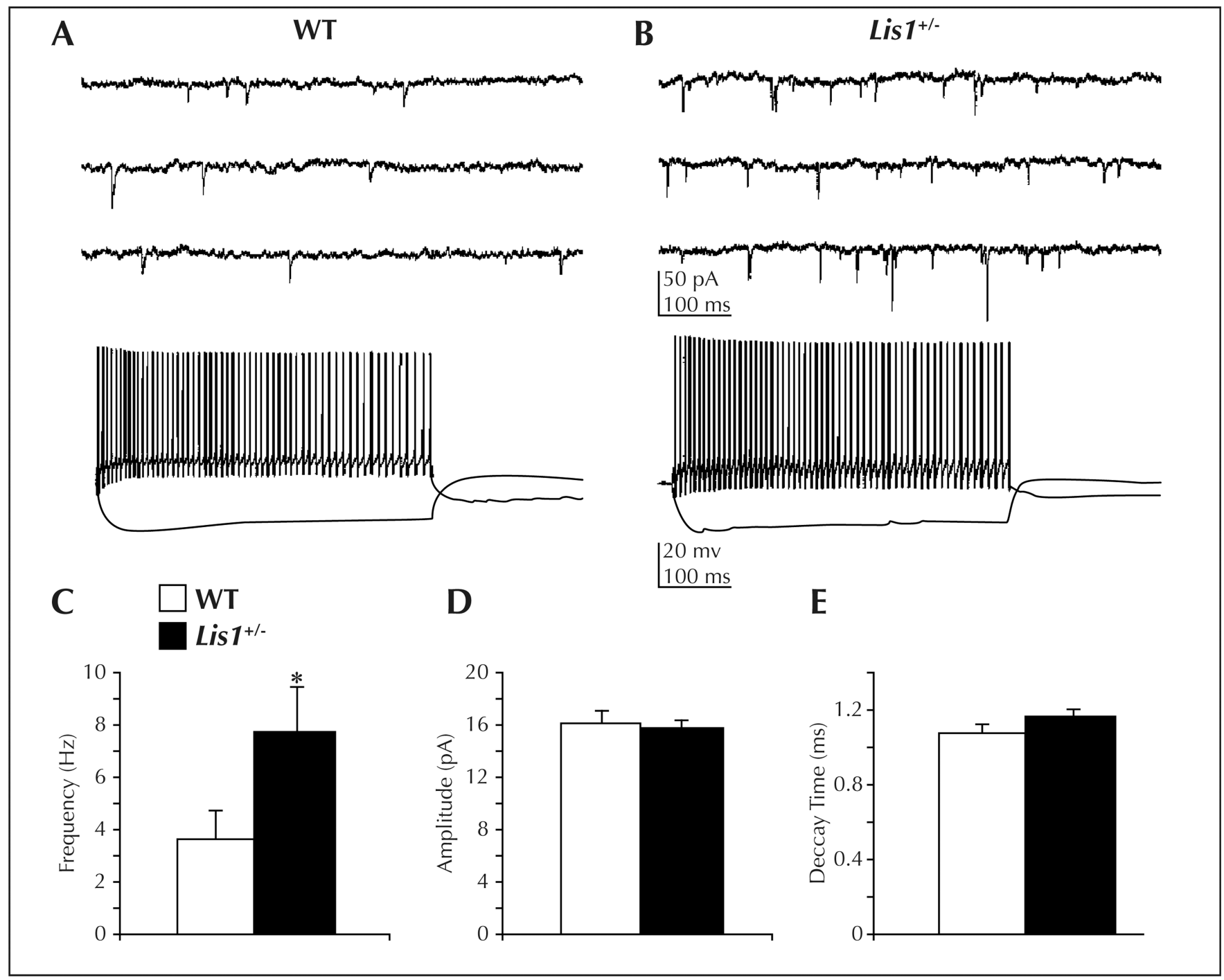

Figure 4.

An increase in excitatory drive onto GABAergic interneurons underlies enhanced activity of inhibitory systems in the hippocampus of Lis $1 \pm$ mice (from Jones and Baraban, 2007). A) Top: whole cell voltage-clamp recording of sEPSCs on a WT interneuron. Bottom: same cell recorded in current-clamp mode. B) Top: sEPSC recording from a Lis $1 \pm$ interneuron. Bottom: same cell recorded in current-clamp mode. C) Mean sEPSC frequency is increased in Lis $1 \pm$ interneurons ( $\mathrm{p}<0.05$, Student's t-test). D) Mean sEPSC amplitude is similar between WT and mutant mice. E) Mean sEPSC decay time is similar between WT and mutant mice. 


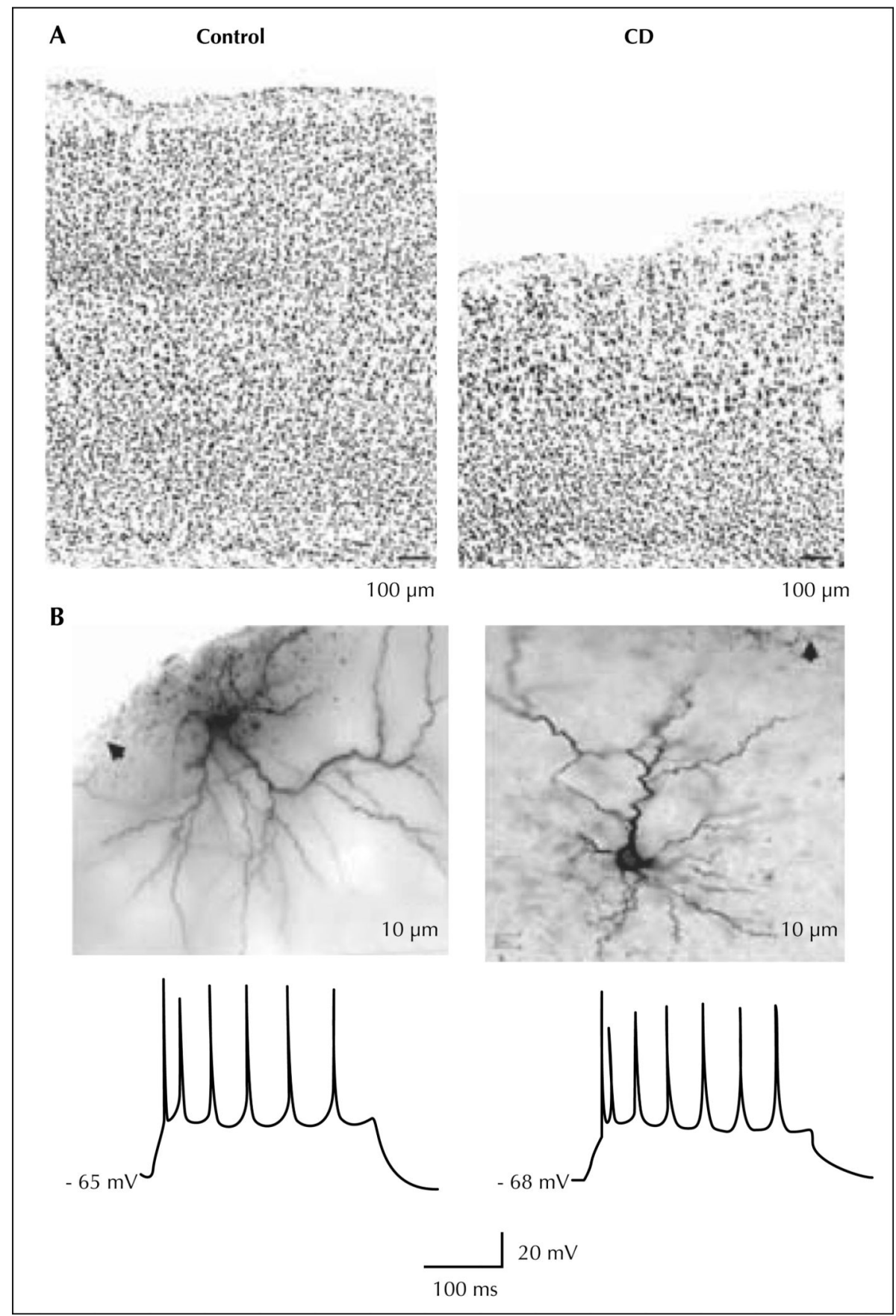

Figure 5.

In utero irradiated rat model of cortical dysplasia. Pyramidal cells from the dysplastic cortex (CD) are spatially disorganized and dysplay a regular spiking pattern to suprathreshold current pulses. A) Low power photomicrographs of coronal sections of control (left) and dysplastic (right) cortex with cresyl violet staining. B) Photomicrographs (upper) show two biocytin-stained pyramidal cells from dysplastic cortex. Arrows point to pia. Traces (lower) show spike patterns of these two cells to depolarizing current injection (300 ms, $300 \mathrm{pA}$ ). 